Gefässchirurgie 2014 • 19:10-12

DOI 10.1007/s00772-013-1285-7

Online publiziert: 10. Februar 2014

(c) Springer-Verlag Berlin Heidelberg 2014

\title{
TASC (Transatlantic Inter-Society Consensus Document) III und ISVaC (International Standards for Vascular Care)
}

ESVS, SVS und WFVS erkennen an,

Das ursprüngliche TASC(Transatlantic Inter-Society Consensus for the Management of Peripheral Artery Disease)Dokument wurde im Jahr 2000 im Journal of Vascular Surgery [1] und im European Journal of Vascular Surgery [2] publiziert. Das erste Update, TASC II, wurde 2007 veröffentlicht [3]. Für 2011 war ein weiteres Update geplant, TASC IIb. Doch wegen mangelnder Übereinstimmung in zahlreichen Aspekten wurde dieses Vorhaben nicht umgesetzt.

Daraufhin nahm sich die TASC-Gruppe eine neue Version vor, es sollte das TASC-III-Dokument werden. Die European Society for Vascular Surgery (ESVS) und die Society for Vascular Surgery (SVS) konnten sich allerdings mit den vorgeschlagenen Regeln für das TASCSteering-Komittee, Art und Umfang der Industriebeteiligung sowie den Regeln zum Ausschluss von Interessenkonflikten nicht einverstanden erklären.

Im September 2013 fand in Budapest eine Konferenz von SVS, ESVS und der World Federation of Vascular Societies (WFVS) statt. In diesem Rahmen einigten sich die drei Fachgesellschaften darauf, sich aus dem TASC-III-Prozess zurückzuziehen.

Dieser Beitrag ist eine Übersetzung der englischen Originalpublikation: Statement from the European Society of Vascular Surgery and the World Federation of Vascular Surgery Societies: Transatlantic Inter-Society Consensus Document (TASC) III and International Standards for Vascular Care (ISVaC). European Journal of Vascular and Endovascular Surgery. Available online 28 November 2013, ISSN 1078-5884, http://dx.doi.org/10.1016/j.ejvs.2013.11.009.

Mit freundl. Genehmigung von Elsevier dass höchste internationale Standards für die gefäßchirurgische Versorgung definiert, generiert und implementiert werden müssen.

Überall auf der Welt ist die Gefäßchirurgie sowohl für die akute als auch für die langfristige Versorgung von Patienten mit peripherer arterieller Verschlusskrankheit das primär verantwortliche Fach. Das Spektrum der gefäßchirurgischen Expertise reicht von konservativen über endovaskuläre bis hin zu offen-chirurgischen Therapieoptionen. In der Regel wird ein Patient für den Rest seines Lebens behandelt und oft bedarf er aller drei Therapiemodalitäten. Für die Versorgung dieser Patienten weltweit gültige Standards zu setzen, muss vornehmlich in der Hand von $G$ efäßchirurgen verbleiben.

Um diese Ziele zum Wohl der Patienten zu erreichen, haben sich die drei Gesellschaften vorgenommen, unter Beteiligung anderer Disziplinen internationale Versorgungsstandards für alle Aspekte peripherer Gefäßerkrankungen zu generieren und zu publizieren. Die Entwicklung dieser Standards wird evidenzbasiert sein und frei von Einflüssen der Industrie.

Als erster Schritt wurde eine „overview group “ benannt, die sich aus jeweils einem Vertreter der o.g. Fachgesellschaften zusammensetzt und 2 Ziele verfolgt:

1. Prozesse für das Setting, die Definition und Einrichtung von Standards festzulegen, sowie

2. festzulegen, wie Kliniker und Fachgesellschaften anderer Disziplinen einzubeziehen sind.

Als erster Schritt sollen Themen („topics“) und die Leitung und Zusammensetzung der einzelnen Arbeitsgruppen festgelegt werden. Dabei besteht eine direkte Verantwortlichkeit für die Organisation und Generierung internationaler Standards.

Zur „three-man group“ gehören Prof. Andrew Bradbury, Birmingham, England, Prof. Philippe Kolh, Lüttich, Belgien, und Prof. Michael Conte, San Francisco, USA.

Für die drei Gesellschaften: ${ }^{1}$

- Jean-Baptiste Ricco, Präsident ESVS

- Simon Parvin, Generalsekretär ESVS

- Julie Ann Freischlag, Präsident SVS

- Michel Makaroun, Sekretär SVS

- Martin Veller, Präsident WFVS

- Jan Brunkwall, Sekretär WFVS

Unter der aktiven Mitwirkung von:

- Prof. John Wolfe, London, England

- Prof. George Hamilton, London, England

- Dr. John White, Columbia, USA

- Prof. Peter Gloviczki, Rochester, USA

- Prof. Peter Lawrence, Harvard, USA

- Prof. Henrik Sillesen, Kopenhagen, Dänemark

- Prof. Hans-Henning Eckstein, München

- Prof. Robert Fitridge, Adelaide, Australien 
Hier steht eine Anzeige.

黛 Springer 


\section{Literatur}

1. TASC Working Group (2000) Management of peripheral arterial disease (PAD). TASC Working Group. TransAtlantic Inter-Society Consensus (TASC). J Vasc Surg 31:S1-S296

2. TASC Working Group (2000) Management of peripheral arterial disease (PAD). TransAtlantic InterSociety Consensus (TASC). Eur J Vasc Endovasc Surg 19 (suppl A):Si-xxviii, S1-250

3. Norgren L, Hiatt WR, Dormandy JA, Nehler MR, Harris KA, Fowkes FGR, on behalf of the TASC I Working Group (2007) Inter-Society Consensus for the Management of Peripheral Arterial Disease (TASC II) J Vasc Surg 45 (suppl):S5-S67

\section{Boris A. Radeleff (Hrsg.) \\ Angiofibel}

Interventionelle angiographische

Diagnostik und Therapie

Heidelberg: Springer-Verlag 2013, 530 S., 205

Abb., (ISBN 978-3-642-33229-6), 149.99 EUR

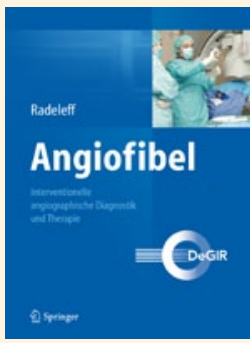

Boris Radeleff hat

mit seinem vorwie-

gend Heidelberger

Autorenteam die

Angiofibel heraus-

gegeben, die sich an

der bewährten Rönt-

genfibel orientiert.

Zunächst als kurze

Arbeitsanweisung für den angiographischen

Alltag bestimmt, hat sich daraus ein über 500

Seiten umfassendes Werk entwickelt.

Im ersten Teil werden ausführlich und praxisnah die Techniken der angiographischen

Diagnostik und Therapie sowie das periinterventionelle Management beschrieben.

Anschließend werden arterielle und venöse

Gefäßeingriffe nach Organregionen geglie-

dert behandelt. Detailliert werden spezifische Embolisationstechniken und Prozeduren erläutert, z.B. die Uterusmyomembolisation sowie die selektive interne Radiotherapie (SIRT). Einen breiten Raum nehmen die Interventionen an der Leber, der Pfortader und dem hepatobiliären System ein. Auch seltene Prozeduren, wie z.B. die Lymphangiographie oder CT-gesteuerte Interventionen bei zystischer Echinokokkose sind mit aufgeführt. Einzig die renale Denervation zur Behandlung der arteriellen Hypertonie ist nicht enthalten und sollte bei der nächsten Auflage ergänzt werden.

Beim Lesen merkt man sofort, dass die Fibel „aus der Praxis für die Praxis" geschrieben wurde. Die Autoren verraten viele Tipps und Anregungen zur Planung und Durchführung insbesondere von Gefäßinterventionen und zum Komplikationsmanagement. Die einzelnen Kapitel sind zwar eigenständig von verschiedenen Autoren erstellt, enthalten jedoch viele Querverweise zu angrenzenden Kapiteln. Herausgeber und Verlag ist es gelungen, dass sich das Buch formal und inhaltlich einheitlich darstellt und einfach zu lesen ist mit der Möglichkeit eigene Anmerkungen einzufügen.

Die Angiofibel bietet sowohl dem Anfänger als auch dem Fortgeschrittenen eine Möglich- keit zum Erlernen bzw. Verfeinern interventioneller Eingriffe. Der Text ist übersichtlich gegliedert und wird durch die vielen Abbildungen und Graphiken ergänzend veranschaulicht. Klinische Ergebnisse von interventionellen Eingriffen werden eingehend mit Literaturangaben diskutiert. Die Angiofibel schlägt damit eine Brücke zwischen einem praxisorientiertem „Kochbuch" und einem wissenschaftlich orientierten Arbeitsbuch. Sie wird vielen interventionell tätigen Ärzten ein guter Ratgeber sein und sollte in keiner angiographischen Abteilung fehlen.

M. Libicher (Schwäbisch Hall) 